\title{
A Bio-inspired Cementitious Composite for High Energy Absorption in Infrastructural Applications
}

\author{
D.G. Soltan ${ }^{1,2}$, R. Ranade ${ }^{3}$, V.C. Li $^{1,3}$ \\ ${ }^{1}$ University of Michigan, Macromolecular Science and Engineering, ${ }^{2}$ dsoltan@umich.edu \\ ${ }^{3}$ University of Michigan, Department of Civil and Environmental Engineering
}

\begin{abstract}
Taking cues from nature's nacre material, several composite design features have been identified as promoting strength and ductility in an otherwise brittle system. These features are to be adapted to, and evaluated for feasibility on, a scale, and with processing routes, applicable to civil infrastructure in an effort to improve the toughness (energy absorption) of cementitious composites. This study is an investigation of one of those features - the layered composite design - in the engineered cementitious composite (ECC) system. Several layering schemes feasible for an infrastructural material are tested in beam bending comparatively with the monolithic material. The goal is to improve energy absorption via enhancement of flexural strain capacity. The addition of intentionally weak interfaces via precast/cast-in-place hybrid layering gave a $62 \%$ increase in average inelastic flexural toughness. Increasing the number of these interfaces and adding an interfacial waviness mimicking another element of nacre's composite design improved performance by $140 \%$ over the monolithic material by the same metric. These results have implications for cementitious composite design philosophy. Enhancing ductility via layering can improve beam member performance by maximizing energy absorption and thus improving durability and safety. This investigation represents the first module of a cumulative and comprehensive material development of a nacre-inspired, cementitious composite design aimed at optimizing strength, ductility, and toughness.
\end{abstract}

Keywords: bio-inspired, concrete, composite, nacre, cementitious

\section{Introduction}

Concrete's lack of tensile strength makes it susceptible to failure by cracking and rapid degradation in many loading scenarios. The development of strain-hardening cementitious composites has provided a degree of tensile strength and ductility to cementitious infrastructural materials [1,2], but nature suggests we can do better. Nacre, the iridescent material seen inside many seashells, is composed $95 \%$ of brittle calcium carbonate; yet, it exhibits remarkable tensile strength and toughness [3]. In nacre, the key strategy employed by nature to transform the brittle, chalk-like calcium carbonate into a material that can outperform our high-performance concretes, is 'shaping' - the shaping of only a few commonplace raw materials such that structure, rather than simply the material, grants function [4].

Research of nacre over the last decade has identified four principal features of nacre's composite design that generate its characteristic deformation mechanisms and yield its impressive behavior [5]. The hierarchical composite design of nacre can be decomposed into the following four elements in descending order of scale: (1) A layered structure in which (2) tiled, discontinuous tablets of calcium carbonate with (3) a marked waviness on their surfaces, stack snuggly with (4) a thin, interfacial polymer layer between. These features comprise a structure that encourages deformation via tablet sliding and promotes local slip-hardening and composite scale strain-hardening behavior that spreads deformation throughout the material. This allows large amounts of energy absorption, damage tolerance, and suppression of catastrophic fracture. 
Can these features of nacre's structure be adapted to a size scale relevant to civil infrastructure and utilized to the same end? This research ultimately aims to answer that and to develop a cementitious material that fills the performance "whitespace" between ultra-high performance concrete and strain-hardening cementitious composites. As a study of the use and utility of just one of those previously identified key features in an ECC system, this current investigation represents the first module of that development. Herein, the layered structure feature is tested experimentally in the context of cementitious infrastructural materials. Starting simply, the performance of several basic layering schemes in beam bending are compared to that of the uniform, monolithic design; although the synergistic effect of the layer waviness is also investigated in this study, there is no attempt at optimization of this additional design feature.

\section{Experimental Design and Methods}

Beams of 3"x3"x14" dimensions were cast using a variation of the high strength, high ductility ECC (HSHDC) material previously developed by Ranade et al. [6]. Water content of the Ranade et al. material composition was increased to increase flowability and improve mold filling. The Type-H cement : Silica Fume : Silica flour : F110 sand : F60 sand : water : ADVA190 superplasticizer : vinylsiloxane coupling agent : Spectra high tenacity polyethylene fiber ratios are $1.00: 0.250$ : $0.070: 0.285: 0.640: 0.300: 0.016: 0.010: 0.020$ by weight. Five specimen types were cast: "monolithic", "flat layered", "wavy layered", "high interfacial area flat layered", and "high interfacial area wavy layered". Monolithic (M) specimens were simply cast into the mold using HSHDC. Flat layered (FL) specimens were cast using two fully cured, pre-cast 0.5 " 3 " 12 " "coupon" specimens also made using HSHDC. A 0.5" layer of fresh HSHDC paste was cast into the beam mold, on top of which a precast coupon was placed. Then another 0.5 " fresh paste layer was cast and the process was repeated such that a 5layer ( 2 precast, 3 freshly cast) specimen was produced. The wavy layered (WL) specimens were cast in the same way with specially molded coupons with two opposing, large "wavy" surfaces. The high interfacial area (HIA) specimens were produced using thinner, approximately 0.25 " thick, coupon specimens with more of them in each beam. Four thin precast coupons were layered with 0.25 " fresh HSHDC layers between to create a 9-layer beam specimen. This was done with flat and wavy precast layers (HIA FL and HIA WL, respectively). All beam specimens were demolded 2 days after casting, and underwent an accelerated curing process: 7 days in room temperature water bath, 4 days in water bath at elevated temperature (90C), and 2 days drying at 90C. Beams were sectioned into approximately 1.5 "x3"x14" specimens for slow-rate testing using a four-point bending setup. Exact dimensions of each specimen were recorded and used to calculate flexural stress using Equation 1, where $\mathrm{F}$ is load, $\mathrm{L}$ is support span, $\mathrm{b}$ is specimen width, and $\mathrm{d}$ is specimen height. The bending experiments were performed at $17 \pm 1$ day curing age. A 12-inch support span and 4-inch loading span were used. Fixed displacement loading was applied and load and "average third point displacement" were recorded. Average third point displacement is a measurement of displacement at the top two loading support points, positioned 4 inches apart, each at a "third" of the 12 inch span. This is measured by instrument stroke during testing.

$$
\text { Flexural Stress, } \sigma_{\mathrm{f}}=(\mathrm{FL}) /\left(\mathbf{b d}^{2}\right)
$$

Flexural stress versus displacement curves were used for analysis of elastic stiffness, ultimate strength (modulus of rupture), first cracking strength, inelastic displacement, and inelastic flexural toughness. Inelastic displacement is calculated by finding the stroke displacement at the point where the beam stress is $90 \%$ of the max stress (modulus of rupture) of the beam after this max has been reached, and subtracting the displacement at which the first crack in the specimen appeared. This inelastic displacement calculation normalizes the effects of the loading points "settling" onto the slightly uneven surface and slight differences in the preloading of the specimen prior to the test. Inelastic flexural toughness is approximated herein by averaging the first cracking strength and the modulus of rupture and then multiplying by the inelastic displacement previously described. This metric is described graphically in Figure 1. This approximated quantification method was used for simplicity and convenience.

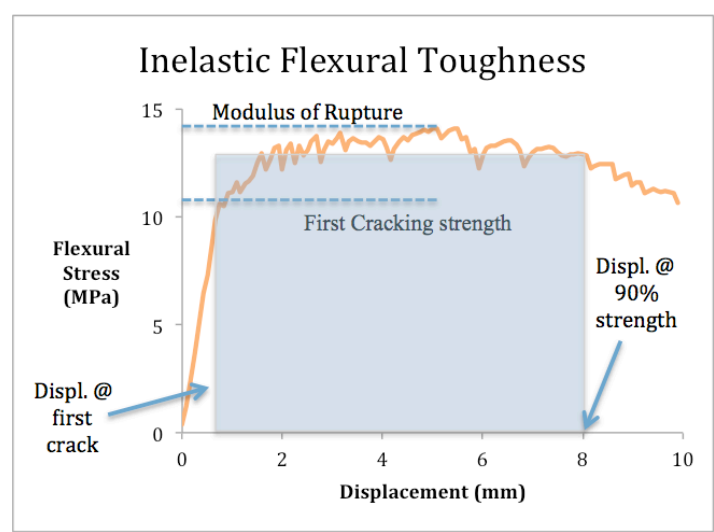

Figure 1: A graphical illustration of the derivation of the "inelastic flexural toughness" metric used in analysis of the beam bending in this study. The shaded area bounded by the first cracking displacement, the displacement at $90 \%$ of the modulus of rupture value, and the average of first cracking strength and modulus of rupture, is taken to be the inelastic flexural toughness. 


\section{Results and Discussion}

The modulus of rupture (MOR) results for each of the seven distinct specimen types are presented in Figure 2(a). All results reported here are averages of 4 specimens for each layering scheme $(n=4)$, with the exception of the WL and HIA WL specimens $(\mathrm{n}=2)$. Comparing the MOR results of M, FL, and HIA FL, it appears that introducing weak interfaces in the beam via layering does little to change the MOR of the beam. Similarly, the interfacial waviness shows no discernable improvement in MOR, when the MOR of M, WL, and HIA WL are compared. The MOR value only denotes the stress at which the beam cannot carry an increasing load, and does not represent a failure to carry load. It is the beam performance after reaching the MOR value that is seen to be affected by the layering schemes. All layering schemes demonstrated significant load bearing capacity past this MOR value, and greater than that of the monolithic scheme. Generally, MOR marked the localization of cracking in the most tensile (bottom) layer, after which load was shared principally among the remaining, less damaged layers. Delamination of layers initiated prior to MOR and continued after MOR. The lower MOR results in this study than those reported in Ranade et al. (25-30 MPa) are attributed to the compositional changes to the material [6].

Effects of the layering schemes become apparent in the comparison of first crack strengths, featured in figure 2(b). First crack strength is associated with the first load drop of the flexural stress versus displacement curve. Here, several trends are observed. First, the use of layers increases the stress at which the first crack occurs by $25 \%$ (FL vs. M). Increasing the number of layers and decreasing their thickness, as in the HIA specimens, further increases the first crack strength an additional $9 \%$ (HIA FL vs. FL). A possible reason behind this observation can be the expected reduction of the flaw size due to the use of thinner layers, but further observations of flaw sizes are needed to support this hypothesis. Secondly, the addition of waviness to the interfacial surface of the layers further increases this first crack strength value by $16.6 \%$ in the 5 -layer specimens and $10.8 \%$ in the 9-layer specimens. The combination of the two factors yields the best consistent performance.

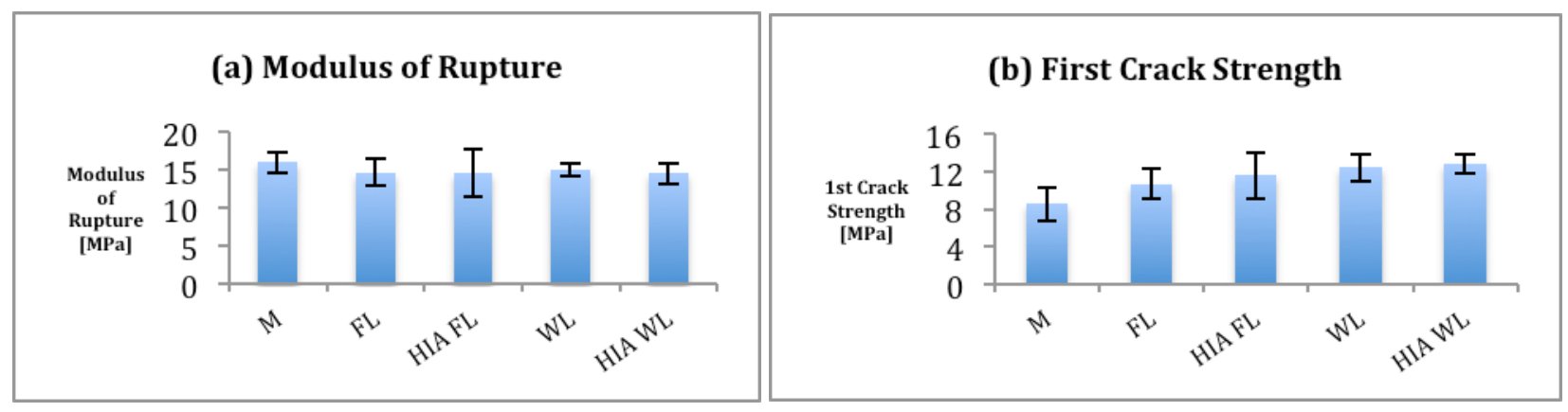

Figure 2: Graphical comparisons of (a) modulus of rupture and (b) first crack strength for each layering scheme.

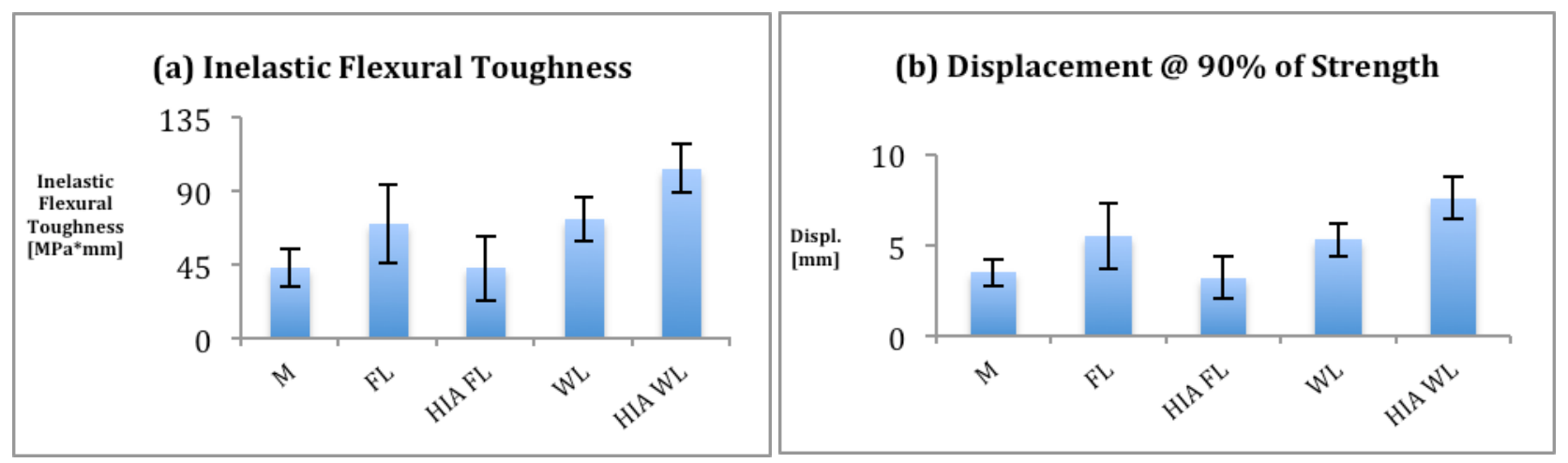

Figure 3: Graphical comparison of the (a) inelastic flexural toughness and (b) the third point, inelastic, vertical displacement at $90 \%$ of the modulus of rupture (MOR) strength for the various layering schemes.

The inelastic flexural toughness (IFT) metric demonstrates the benefits of the various layering schemes. Seen in Figure 3(a), these results show an increase in toughness with the use of the precast/cast-in-place hybrid layering. With modulus of rupture values varying only slightly between schemes, these toughness results largely mirror the beam's ductility represented by the displacement values (Figure 3(b)), one of the factors upon which IFT is based. The introduction of weak interfaces via layering boosted the FL toughness $62 \%$ over that of the monolithic specimens. Increasing the amount of these interfaces with the use of thinner layers and more of them, while adding a distinct wavy pattern to the surfaces, increased the toughness $140 \%$. The HIA FL toughness value appears to be an anomaly; however, the specific behavior of these specimens is not fully captured by this metric. Despite low displacement at $90 \%$ of the MOR value, these specimens exhibit the largest displacement at $\sim 70 \%$ of MOR. Relative to the other schemes, the HIA FL specimens show a large plateau in load capacity post peakstrength, due to the large degree of layer delamination and layer sliding as pictured in Figure 4(a). This plateau occurs at 
$\sim 72 \%$ of MOR, following a drop in strength after reaching the maximum load. Despite not receiving credit for its performance via the IFT metric, the effective ductility of this scheme represents a significant improvement in energy absorption over the monolithic scheme.

It was seen that the addition of interfacial waviness, via specially molded pre-cast coupons with wavy surfaces, improved the beams load carrying capacity at large deformations $(5+\mathrm{mm})$. The layer waviness likely provides resistance to both layer delamination and sliding (the mechanisms responsible for the increased ductility of the layering schemes) via increased initial mechanical bonding between layers and frictional effects after debonding. When the magnitude of this waviness is increased relative to layer thickness (as in the HIA WL scheme), these effects appear to be amplified.
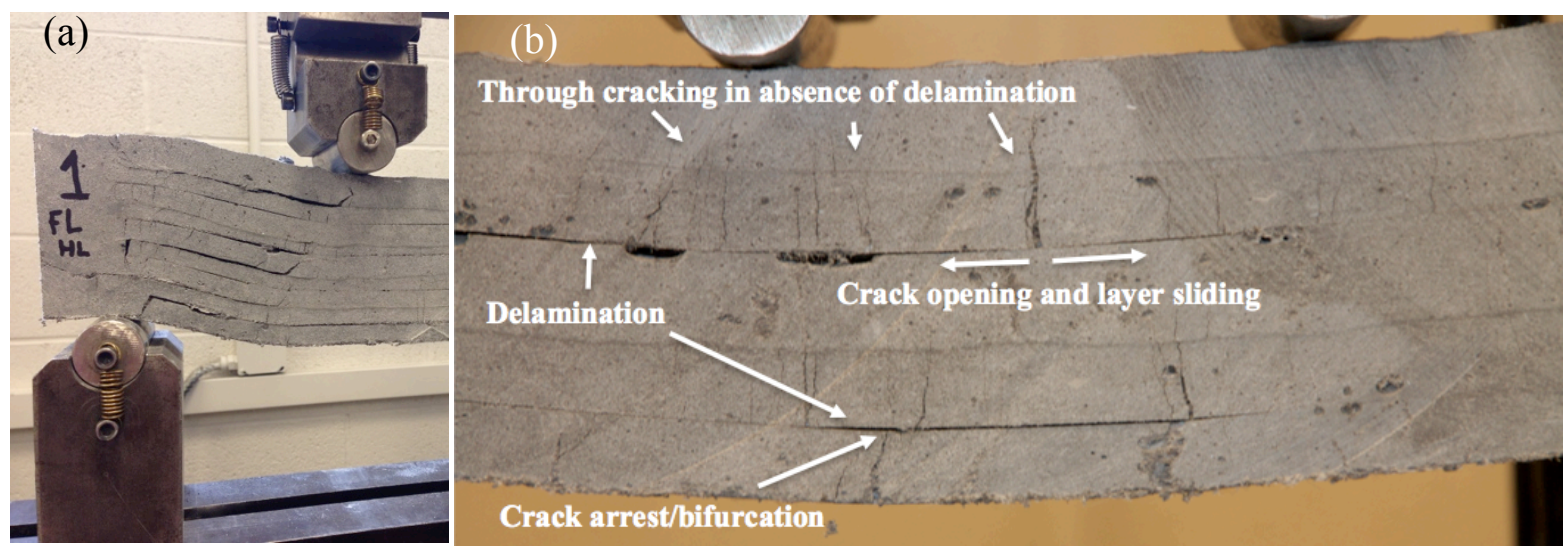

Figure 4: Layer delamination and subsequent sliding as observed in (a) the HIA FL specimens, and (b) the FL specimens. Several energy absorbing mechanisms are highlighted in Figure 4(b).

Figure 4(b) highlights several of the energy absorbing mechanisms observed in the layered specimens. Although only the FL scheme is shown, these energy absorbing mechanisms were observed, to different degrees, in all of the layered specimens. It is important to note that layer delamination is the key for the other mechanisms to occur. Crack arrest, bifurcation, and diffusion (not pictured) are not observed locally where layers appear to remain bonded. This results in through cracking between layers, much like the behavior exhibited in the monolithic case. Extreme delamination (as in the HIA FL case), however, gives toughness values less than those recorded for other cases. Thus, an optimized interlayer bonding, not too strong and not too weak, to give maximal energy absorption is implied. Interfacial bonding was shown to be manipulated by the waviness feature of the precast layers. Delamination did not occur until higher loads in WL and HIA WL specimens as compared to FL and HIA FL specimens, and often much less delamination was observed even after the wavy specimens had been tested to failure. Manipulation of this interfacial bonding could be further controlled via tailoring of waviness parameters (amplitude, period, etc.) and by an additional, thin interfacial layer inspired by nacre's composite design. These elements of design will be investigated and developed in future studies.

\section{Conclusions}

A hybrid precast/cast-in-place layering concept inspired by the seashell material nacre was investigated in the context of infrastructural composite beam design. This composite design feature was shown to increase inelastic flexural toughness, a metric indicative of energy absorption. The effect was enhanced by increasing the amount of the intentionally weak interfaces in the beam member and by using a wavy shape for these interfacial surfaces. Such a finding has significance for design of critical infrastructural members and also presents a novel hybrid precast/cast-in-place construction technique. Future work aims to improve performance via use of additional composite design features inspired by nacre: a second phase interfacial layer and a discontinuous, tablet-like, layer structure. The waviness and layer parameters will also continue to be optimized.

\section{Acknowledgements}

This research has been supported by the University of Michigan MCubed interdisciplinary research seed-funding program. The authors would also like to acknowledge Lafarge (cement), US Silica (sand and ground silica), Elkem (microsilica), Honeywell (PE fiber), and WR Grace (superplasticizer) for providing material samples for this research.

\section{References}

1. Advances in Cement-Based Materials, Proc., Int'l Conference on Advanced Concrete Materials, Stellenbosch, S. Africa, (Nov. 2009), 21-28.

2. V.C. Li, On Engineered Cementitious Composites (ECC), Journal of Advanced Concrete Technology, Vol. 1, No. 3 (2003) 215-230

3. F. Barthelat, and H.D. Espinosa, An investigation of deformation and fracture of nacre - mother of pearl, Exp. Mech. (2007) 47:311-324

4. J. Benyus, Biomimicry and the quest to solve global challenges, $13^{\text {th }}$ annual Peter M. Wege Lecture on Sustainability, Ann Arbor, MI (Mar. 2014)

5. F. Barthelat, J.E. Rim, and H.D. Espinosa, A review of the structure and mechanical properties of mollusk shells-perspectives on synthetic biomimetic materials, Applied Scanning Probe Methods XIII, Springer (2009) 17-44

6. R. Ranade, V.C. Li, M.D. Stults, W.F. Heard, and T.S. Rushing, Composite Properties of High-Strength, High-Ductility Concrete, ACI Materials Journal, Vol. 110, Issue 4, (2013) 413-422 